Research article

\title{
A new ophthalmic formulation containing antiseptics and dexpanthenol: In vitro antimicrobial activity and effects on corneal and conjunctival epithelial cells
}

\author{
Rita Mencucci $^{\mathrm{a}, *, 1}$, Eleonora Favuzza ${ }^{\mathrm{a}, 1}$, Paolo Bottino ${ }^{\mathrm{b}}$, Costanza Mazzantini ${ }^{\mathrm{c}}$, Elisa Zanotto ${ }^{\mathrm{b}}$, \\ Domenico E. Pellegrini-Giampietro ${ }^{c}$, Elisa Landucci ${ }^{c}$

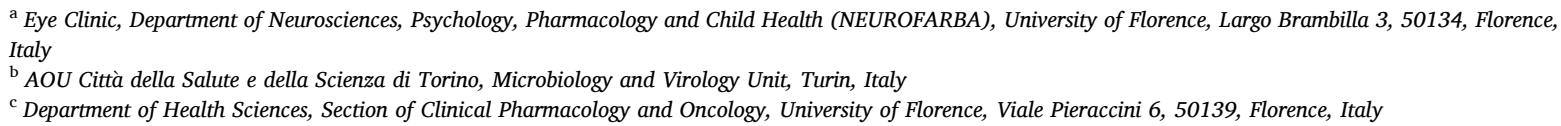

\section{A R T I C L E I N F O}

\section{Keywords:}

Antiseptics

Hexamidine

Dexpanthenol

Polyhexamethylene biguanide

PHMB

EDTA

Corneal epithelial cells

Conjunctival epithelial cells

\begin{abstract}
A B S T R A C T
Antibiotic resistance is increasing even in ocular pathogens, therefore the interest towards antiseptics in Ophthalmology is growing. The aim of this study was to analyze the in vitro antimicrobial efficacy and the in vitro effects of an ophthalmic formulation containing hexamidine diisethionate $0.05 \%$, polyhexamethylene biguanide (PHMB) $0.0001 \%$ disodium edetate (EDTA) $0.01 \%$, dexpanthenol 5\% and polyvinyl alcohol $1.25 \%$ (Keratosept, Bruschettini, Genova, Italy) on cultured human corneal and conjunctival cells. The in vitro antimicrobial activity was tested on Staphylococcus aureus, Methicillin-Resistant Staphylococcus aureus (MRSA), Pseudomonas aeruginosa, Streptococcus pneumoniae, Streptococcus pyogenes and Streptococcus mitis. For each microbial strain $10 \mu \mathrm{L}$ of a 0.5 MacFarland standardized bacterial inoculum were incubated at $25{ }^{\circ} \mathrm{C}$ with $100 \mu \mathrm{L}$ of ophthalmic solution for up to $6 \mathrm{~h}$. After different periods of time, samples were inoculated on blood agar with $5 \%$ sheep blood. Moreover, a 0.5 MacFarland bacterial inoculum was seeded in triplicate on Mueller-Hinton Agar or on Mueller-Hinton Fastidious Agar; then a cellulose disc soaked with $50 \mu \mathrm{L}$ of ophthalmic solution was applied on the surface of agar and plates were incubated for $18 \mathrm{~h}$ at $37{ }^{\circ} \mathrm{C}$, in order to evaluate the inhibition of bacterial growth around the disc. Human corneal and conjunctival epithelial cells in vitro were incubated for 5, 10 and 15 min with Keratosept or its components. The cytotoxicity was assessed through the release of cytoplasmic enzyme lactate dehydrogenase (LDH) into the medium immediately after exposure to the drugs; the 3-(4,5-dimethylthiazol-2-yl)-2,5-diphenyltetrazolium bromide (MTT) assay was performed to evaluate the metabolic cell activity. Our results show that Keratosept ophthalmic solution gave an average logarithmic (log) reduction of bacterial load of $2.14 \pm 0.35$ within $6 \mathrm{~h}$ of exposure (p-value $<0.05$ versus control saline solution). On agar plates, all microbial strains, excluding P. Aeruginosa, showed an inhibition zone of growth around the Keratosept-soaked discs. Keratosept and its components after 5 and $10 \mathrm{~min}$ did not show any cytotoxic effect on cultured corneal and conjunctival cells, and only after $15 \mathrm{~min}$ a significant reduction of cell viability and an increase of cytotoxicity compared to control (vehicle) was seen; dexpanthenol 5\% and polyvinyl alcohol accelerated the wounding of corneal cells in vitro. In conclusion, Keratosept showed good antimicrobial activity on the tested strains; the ophthalmic solution and its components were safe and non-toxic for the corneal and conjunctival epithelial cells for 5 and $10 \mathrm{~min}$ at the concentrations analyzed, and dexpanthenol $5 \%$ and polyvinyl alcohol promoted the wounding of corneal cells.
\end{abstract}

\footnotetext{
* Corresponding author. Department of Neuroscience, Psychology, Pharmacology and Child Health (NEUROFARBA), Eye Clinic, University of Florence Largo Brambilla 3, 50134, Florence, Italy.

E-mail addresses: rita.mencucci@unifi.it (R. Mencucci), elefavuzza@gmail.com (E. Favuzza), paolo.bottino@unito.it (P. Bottino), costanza.mazzantini@unifi.it (C. Mazzantini), elisa.zanotto@unito.it (E. Zanotto), domenico.pellegrini@unifi.it (D.E. Pellegrini-Giampietro), elisa.landucci@unifi.it (E. Landucci).

1 These authors contributed equally to this work.
} 


\section{Introduction}

Antimicrobial resistance to antibiotics is one of the biggest public health challenges of our time and it has been continuously increasing in recent years, even in ocular pathogens (Asbell and DeCory, 2018; Bertino, 2009; Holland et al., 2014). As in all human infections, the rise of bacterial resistance can also be of concern in ophthalmology, leading to unsuccessful treatment of sight-threatening infections (Asbell and DeCory, 2018; Bertino, 2009; Holland et al., 2014).

Since the overuse of antibiotics in the treatment of ocular infectious diseases has led to the appearance of multidrug-resistant bacterial strains (Asbell and DeCory, 2018), the interest towards antiseptics is growing. In ophthalmology, the role of topical antibiotics in the prophylaxis of post-surgical endophthalmitis, especially in intravitreal injections, is under debate because their perioperative use can increase the resistance of conjunctival flora but has no proven added effectiveness in preventing endophthalmitis if combined with povidone iodine antisepsis (Grzybowski et al., 2017).

Moreover, due to their wide spectrum of activity (multi-drug resistant bacteria, methicillin-resistant staphylococcus aureus, viruses, protozoa, fungi) (Grzybowski et al., 2018; Kaehn, 2010; Maycock and Jayaswal, 2016), and the few reports on reduced microbial susceptibility which currently only concern chlorhexidine (Horner et al., 2012; Kampf, 2016), the use of antiseptics has been proposed for the treatment of viral conjunctivitis, mild bacterial conjunctivitis, or even more severe infections (such as keratitis) in developing countries, where the availability of antibiotics is often limited by costs (Grzybowski et al., 2018; Isenberg et al., 2017). Regarding Acanthamoeba keratitis, the only available treatment are the antiseptics biguanides (PHMB 0.02\%, and chlorhexidine $0.02 \%$ ) and diamidines (hexamidine $0.1 \%$ or propamidine 0.1\%) (Carrijo-Carvalho et al., 2017; Maycock and Jayaswal, 2016).

However, antiseptics may be toxic for the ocular surface epithelia, leading to corneal epithelial defects or damage, especially at high concentrations (Shibata et al., 2014). Therefore, there is an increasing interest in topical formulations containing antiseptics with a wide antimicrobial spectrum, but with a lower tendency to damage the ocular surface. Recently, an ophthalmic solution containing antiseptics, such as hexamidine diisethionate $0.05 \%$, PHMB $0.0001 \%$, disodium edetate (EDTA) $0.01 \%$, and healing agents, such as dexpanthenol (D-panthenol) $5 \%$ and polyvinyl alcohol $1.25 \%$, has been launched on the market in Italy (Keratosept, Bruschettini, Genova, Italy). The purposes of our study were to evaluate the antimicrobial activity of this product on isolates of Staphylococcus aureus, Methicillin-Resistant Staphylococcus aureus (MRSA), Pseudomonas aeruginosa, Streptococcus pneumoniae, Streptococcus pyogenes and Streptococcus mitis, that are among the most frequent causes of ocular infections (Asbell and DeCory, 2018; Garg et al., 2017; Green et al., 2008; Han et al., 1996; Teweldemedhin et al., 2017) and to analyze in vitro the effects of this solution and of its components on cultured human corneal and conjunctival cells.

\section{Materials and methods}

\subsection{In vitro antimicrobial activity of the ophthalmic solution}

The antimicrobial activity of the ophthalmic solution was evaluated using a modified protocol from the International Organization for Standardization- ISO 14729 guidelines (International Organization for Standardization, 2001).

Staphylococcus aureus, Methicillin-Resistant Staphylococcus aureus (MRSA), Pseudomonas aeruginosa, Streptococcus pneumoniae, Streptococcus pyogenes and Streptococcus mitis (of the Viridans Group Streptococci, VGS) were used in this study. All the microbial strains were obtained from the collection at the microbiology laboratory of the University Hospital "Città della Salute e della Scienza di Torino", Turin Italy. The isolates of MRSA were resistant to fluoroquinolones and aminoglycosides, while the other bacteria were susceptible to all antimicrobial agents routinely tested. For each species 5 different isolates in triplicates were tested.

For each microbial strain a 0.5 MacFarland standardized bacterial inoculum $\left(1.5 \times 10^{8}\right.$ colony-forming unit $\left./ \mathrm{mL}\right)$ was prepared in sterile phosphate buffered saline solution, then $10 \mu \mathrm{L}$ of inoculum were incubated at $25^{\circ} \mathrm{C}$ with $100 \mu \mathrm{L}$ of ophthalmic solution for up to $6 \mathrm{~h}$. At the defined time points of 10,30 and $45 \mathrm{~min}, 1,2,4,6 \mathrm{~h}, 10 \mu \mathrm{L}$ of solution were removed and added to $100 \mu \mathrm{L}$ of neutralizing broth (Becton Dickinson, USA); then, $10 \mu \mathrm{L}$ of samples were inoculated on blood agar with $5 \%$ sheep blood (Becton Dickinson, USA), plating each sample in triplicate. The numbers of viable bacteria in the samples were determined by plate counts of colony-forming units (CFUs) after serial dilutions following an overnight incubation at $37^{\circ} \mathrm{C}$. A sterile isotonic solution, without antiseptics, was used as a control.

In order to evaluate the ability of bacteria to grow on agar-nutrient plates in the presence of ophthalmic solution, a 0.5 MacFarland bacterial inoculum was seeded in triplicate on Mueller-Hinton Agar (Becton Dickinson, USA) for non-fastidious bacteria ( $S$. aureus, MRSA, $P$. aeruginosa) and on Mueller-Hinton Fastidious Agar (Becton Dickinson, USA) for fastidious bacteria ( $S$. pneumoniae, $S$. pyogenes and $S$. mitis). Then a cellulose disc soaked with $50 \mu \mathrm{L}$ of ophthalmic solution was applied on the surface of the agar and plates were incubated for $18 \mathrm{~h}$ at $37^{\circ} \mathrm{C}$, in order to evaluate the inhibition of bacterial growth around the disc.

\subsubsection{Statistical analysis}

Data are presented as log reduction in CFU per $\mathrm{mL}(\mathrm{Log} \mathrm{CFU} / \mathrm{mL})$ for each microorganism at each exposure time. The $\mathrm{Log} \mathrm{CFU} / \mathrm{mL}$ reduction of control was obtained from the overall mean of all tested strains. Differences between Log reductions were analyzed by using a two-tailed Student $t$-test and a p-value $<0.05$ was considered significant.

\subsection{In vitro effects of the ophthalmic solution and its components on cultured human corneal and conjunctival cells}

\subsubsection{Materials}

Human corneal epithelial cells (HCE-2) [50B1] (ATCC CRL-11135) were purchased from ATCC company (American Type Culture Collection, PO Box 1549 Manassas, VA, 20108, USA). Human conjunctival epithelial cells (HConEC) and corneal epithelium cell medium consisting of the basal medium and corneal epithelial cell growth supplement (CEpiCGS) were provided by Innoprot (Derio, Bizkaia, Spain). Keratinocyte serum-free medium, bovine pituitary extract (BPE) and epidermal growth factor (EGF) were purchased from Gibco-BRL (San Giuliano Milanese, MI, Italy). Hydrocortisone, insulin, fibronectin, bovine collagen type I and bovine serum albumin (BSA) penicillin/ streptomycin $(\mathrm{P} / \mathrm{S})$, bovine fetal serum and poly-L-lysine, benzalkonium chloride (BAK) and 3-(4,5-dimethylthiazol-2-yl)-25-diphenyltetrazolium bromide (MTT) were obtained from Sigma (St Louis, MO, USA). The Cytotoxicity detection kit (lactate dehydrogenase, LDH) was obtained from Roche Diagnostics (Basel, Switzerland).

Keratosept and all its constituents were a kind gift from Bruschettini srl (Genova, Italy).

\subsubsection{Human corneal epithelial cells (HCE-2)}

Human corneal epithelial cells (HCE-2) were kept in culture in keratinocyte serum-free medium supplemented with $0.05 \mathrm{mg} / \mathrm{mL} \mathrm{BPE,} 5$ $\mathrm{ng} / \mathrm{mL}$ EGF, $500 \mathrm{ng} / \mathrm{mL}$ hydrocortisone and $0.005 \mathrm{mg} / \mathrm{mL}$ insulin. The cells were incubated at $37^{\circ} \mathrm{C}$ in an atmosphere of humidified air and $5 \%$ $\mathrm{CO}_{2}$, and then seeded on $75 \mathrm{~cm}^{2}$ flasks precoated with a mixture of 0.01 $\mathrm{mg} / \mathrm{mL}$ fibronectin, $0.03 \mathrm{mg} / \mathrm{mL}$ bovine collagen type I and $0.01 \mathrm{mg} / \mathrm{mL}$ BSA.

The keratinocyte serum-free medium was changed twice a week. Once confluence was reached, the cells were split with a 1:3 proportion into other flasks previously treated. 


\subsubsection{Human conjunctival epithelial cells (HConEC)}

Human conjunctival epithelial cells (HConEC) provided by Innoprot are isolated from human conjunctiva. The cells were kept in culture in conjunctival epithelial cell medium consisting of the basal medium with the addition of the conjunctival epithelial cell growth supplement (CEpiCGS), penicillin/streptomycin (P/S) and bovine fetal serum, in an atmosphere of humidified air and $5 \% \mathrm{CO}_{2}$ at $37{ }^{\circ} \mathrm{C}$. The cells were plated in $75 \mathrm{~cm}^{2}$ flasks previously treated for $1 \mathrm{~h}$ with a solution of poly-Llysine 1:10 in PBS. The culture medium was changed twice a week. Once the confluence was reached, the cells were split with a 1:3 proportion in other previously treated flasks.

\subsubsection{Incubation with drugs}

Human corneal and conjunctival epithelial cells were resuspended and seeded into 24-well plates (approximately $4 \times 10^{-4}$ cells $/ \mathrm{cm}^{2}$ ). When they reached approximately a $70-80 \%$ confluence, the culture medium was removed and the cells were exposed to Keratosept or to all the individual constituents of the formulation (polyvinyl alcohol 1.25\%, D-panthenol 5\%, hexamidine diisethionate 0.05\%, PHMB $0.0001 \%$, methyl sulfone dimethyl sulfone $1.25 \%$, EDTA $0,01 \%$ ) dissolved in the Keratosept vehicle, which consists in a buffer solution (potassium phosphate $0.04 \%$, dibasic sodium phosphate $0.2 \%$ and $\mathrm{H}_{2} 0$ ). The vehicle was used as positive control and BAK $0.01 \%$ for $2 \mathrm{~h}$ was used as negative control for maximum cell death.

Corneal and conjunctival epithelial cells were incubated with drugs in different concentrations (dilution 1:1 and 1:10) and for 3 different periods $(5,10$ and $15 \mathrm{~min})$.

\subsubsection{Analysis of cell viability: MTT assay}

The viability of the conjunctival and corneal epithelial cells exposed for 3 different periods $(5,10,15 \mathrm{~min})$ and in different dilutions (1:1 and $1: 10$ ) to all the individual constituents of Keratosept and to the complete formulation, was evaluated by MTT assay.

The cells were seeded in 24-well plates and maintained in culture. All the drugs were incubated in different concentrations for different periods in the vehicle. Part of the medium of each well was taken and stored for the LDH assay. The cells were incubated with MTT at a concentration of $1 \mathrm{mg} / \mathrm{mL}$ (Piazzini et al., 2019). After removing the MTT solution, dymethyl sulfoxide (DMSO) was added to the wells to dissolve the formation, and the absorbance of MTT was read at 550 and $690 \mathrm{~nm}$. The vehicle was used as a positive control. Cell viability was expressed as a percentage of the cells incubated only in the vehicle in the corresponding exposure period.

\subsubsection{Evaluation of cell death: $L D H$ assay}

Damage in the human corneal and conjunctival epithelial cells was quantitatively evaluated by measuring the amount of the soluble cytosolic enzyme LDH released from injured cells into the extracellular fluid, 5,10 and $15 \mathrm{~min}$ after exposure to the drugs, using the LDH kit, as previously described for mix cortical cells (Landucci et al., 2019).

The LDH level corresponding to complete cell death was determined for each experiment by assaying sister cultures exposed to BAK $0.01 \%$ for $2 \mathrm{~h}$. Background LDH release was determined in control cultures not exposed to drugs, and was subtracted from all experimental values. The resulting values correlated linearly with the degree of cell loss estimated at the observation of cultures under phase-contrast optics.

\subsubsection{Analysis of corneal wound healing}

Human corneal epithelial cells were used for re-epithelialization studies. Double insert wells of biocompatible silicone (width $\mathrm{x}$ length $\mathrm{x}$ height $=8.4 \mathrm{~mm} \times 8.4 \mathrm{~mm} \times 5 \mathrm{~mm}$ ), with a growth size of $0.22 \mathrm{~cm}^{2}$ and a $70 \mu \mathrm{L}$ plating volume for each well, from Ibidi (Lochhamer Schlag, Gräfelfing, Germany) were used for all epithelialization tests. The cells were seeded on the inserts and after $24 \mathrm{~h}$ the inserts were removed leaving a space of $420 \mu \mathrm{m}$ between the two layers of cells, an identical size for each experiment. Then, D-panthenol 5\% and polyvinyl alcohol
$1.25 \%$ were dissolved in the culture medium and corneal cell monolayers were incubated for 24 and $48 \mathrm{~h}$ to test re-epithelialization in comparison with cell inserts exposed to the culture medium alone (control). The extent of healing was evaluated qualitatively in a blind fashion and photographed by an inverted phase-contrast microscope (Olympus IX-50; Solent Scientific, Segensworth, UK) at 10X magnification.

\subsubsection{Statistical analysis}

Data are presented as mean \pm standard error of the mean (SEM) of $n$. experiments. The statistical significance of differences in MTT and LDH release were analyzed using one-way ANOVA with Dunnett's post-hoc test for multiple comparisons. All statistical calculations were performed using GraphPad Prism version 5 for Windows (GraphPad Software, San Diego, CA, USA). A p-value $<0.05$ was considered significant.

\section{Results}

\subsection{In vitro antimicrobial activity of the ophthalmic solution}

The Keratosept ophthalmic solution showed an antiseptic spectrum of activity and gave an average log reduction of $2.14 \pm 0.35$ within $6 \mathrm{~h}$ of exposure, significantly higher than the control solution (p-value $<0.05$ ), although individual $\log$ reductions varied between microorganisms (Fig. 1). Log reductions of the fastidious species (Streptococcus pneumoniae, Streptococcus mitis and Streptococcus pyogenes) were significantly marked within $2 \mathrm{~h}$ of exposure to the ophthalmic solution (p-value $<$ 0.05 vs control for all comparisons). Especially for Streptococcus pneumoniae and Streptococcus mitis a $\log$ reduction of $2.40 \pm 0.39$ and $2.00 \pm$ 0.87 occurred at $30 \mathrm{~min}$ of exposure (Fig. 1).

Of the non-fastidious species (Staphylococcus aureus, MRSA and Pseudomonas aeruginosa), Staphylococcus aureus showed a log reduction of $1.66 \pm 0.26$ after $6 \mathrm{~h}$ of exposure to the ophthalmic solution, while for MRSA the log reduction was $2.32 \pm 0.59$. However, the difference between the two strains was not significant (p-value $>0.05$ ). The log reduction was especially marked for Pseudomonas aeruginosa within 45 min of exposure $(2.08 \pm 0.34)$.

All the microbial strains, except $P$. aeruginosa, after an overnight incubation on agar plates, showed an inhibition zone of growth around the Keratosept-soaked discs, with a mean diameter of $17.27 \pm 4.37 \mathrm{~mm}$ (Table 1).

\subsection{Evaluation of the effects of Keratosept and its constituents on corneal and conjunctival epithelial cells using LDH and MTT assay}

Using LDH as a quantitative cytotoxicity index, we examined the extent of human corneal epithelial cell death. The maximum degree of cell death was produced in our culture system by incubation for $2 \mathrm{~h}$ with $0.01 \%$ BAK, which produced a maximum release of LDH.

After 5 and 10 min of incubation, Keratosept and all its components displayed negligible corneal cell death (Fig. 2 A, B); Keratosept and some of its components, such as EDTA and methyl sulfone dimethyl sulfone, began to be toxic only after 15 min (Fig. 2 C).

The viability of corneal epithelial cells after exposure to Keratosept and its components is shown in Fig. 3. After 5 and 10 min exposure no significant changes in cell viability measured by MTT assay were detected except for BAK 0.01\% (Fig. 3 A, B). However, after 15 min Keratosept, EDTA and methyl sulfone dimethyl sulfone significantly decreased the cell viability to $21 \%, 28 \%$ and $25 \%$ of the vehicle value, respectively. (Fig. $3 \mathrm{C}$ ).

We also tested the Keratosept formulation on the human conjunctival epithelial cells.

At all the 3 incubation periods, Keratosept and all its components caused negligible cell death, like the vehicle (Fig. 4 A, B, C).

Regarding the MTT assay, we observed a reduction of cell viability with Keratosept at $10 \mathrm{~min}$ and with Keratosept, hexamidine 


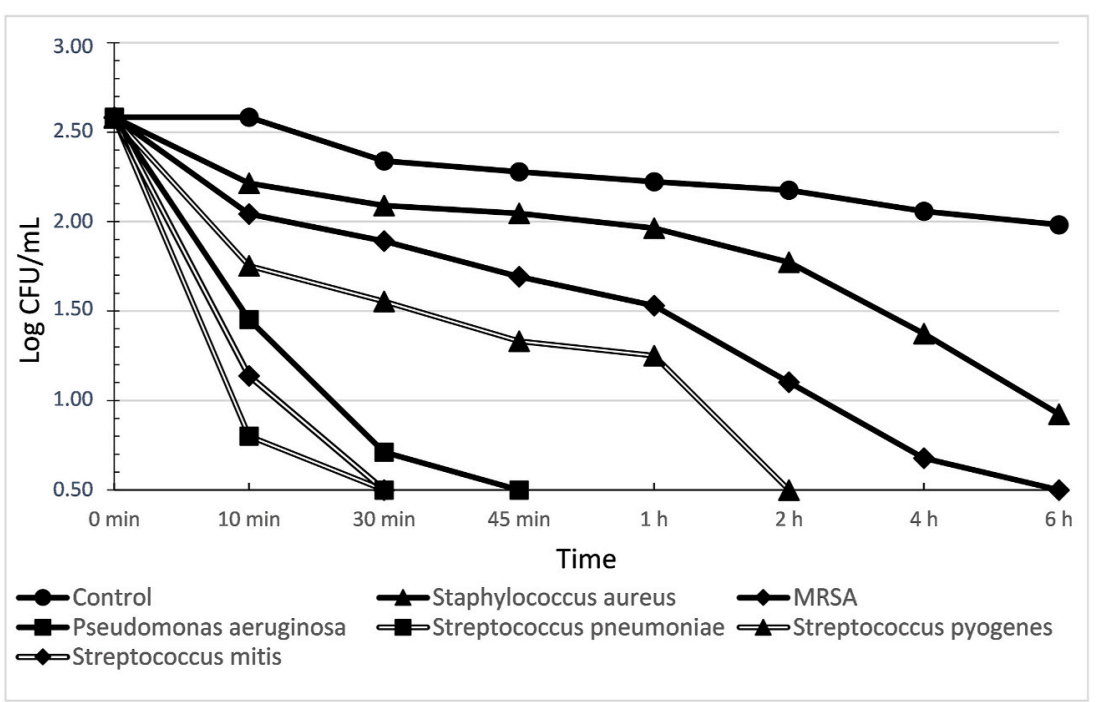

Fig. 1. Antimicrobial activity of Keratosept ophthalmic solution expressed as log CFU/mL reduction in relationship to exposure time. CFU $=$ colony-forming unit, MRSA $=$ Methicillin-resistant Staphylococcus aureus, $\min =$ minutes, $\mathrm{h}=$ hours.

Table 1

Microbial growth on agar plates in presence of Keratosept ophthalmic solution.

\begin{tabular}{ll}
\hline Organism & Growth inhibition at $18 \mathrm{~h}$ \\
\hline Staphylococcus aureus & Presence of inhibition \\
MRSA & Presence of inhibition \\
Pseudomonas aeruginosa & Absence of inhibition \\
Streptoccocus pneumoniae & Presence of inhibition \\
Streptococcus mitis & Presence of inhibition \\
Streptococcus pyogenes & Presence of inhibition \\
\hline
\end{tabular}

MRSA: Methicillin-Resistant Staphylococcus aureus.

diisethionate and EDTA at $15 \mathrm{~min}$. Conversely, the exposure for $5 \mathrm{~min}$ to polyvinyl alcohol and D-panthenol induced a significant increase in the level of MTT assay (Fig. 5).

Finally, we tested Keratosept and all its components diluted 1:10 on human corneal (Fig. 6 A, B) and on conjunctival (Fig. 6 C, D) epithelial cells for $15 \mathrm{~min}$. Keratosept and all its single components showed no signs of toxicity at dilution 1:10 in comparison with BAK $0.01 \%$ (after 2 $\mathrm{h}$ of exposure) (Fig. 6, A and C) and showed no alteration of cell viability
(Fig. 6, B and D).

\subsection{Corneal wound healing}

In human corneal epithelial cells incubated only in culture medium (the control), the analysis of the corneal wound area revealed that the wound was completely covered after $48 \mathrm{~h}$; when the cells were exposed to a combination of polyvinyl alcohol $1.25 \%$ and D-panthenol $5 \%$, the gap was completely covered in $24 \mathrm{~h}$ (Fig. 7).

\section{Discussion}

Increasing trends in antibiotic resistance in ocular infections, especially widespread fluoroquinolone non-susceptibility (Asbell and DeCory, 2018; Bertino, 2009; Holland et al., 2014), have enhanced the role of antiseptics in the prevention of infection after ocular surgery (Grzybowski et al., 2017). Nowadays, povidone-iodine (PVI) and chlorhexidine represent the elective antiseptics in ophthalmology. However, a reduced susceptibility to chlorhexidine of MRSA and other staphylococci was reported by Horner et al. (2012), discouraging its indiscriminate use in the absence of efficacy data. For these reasons,
A

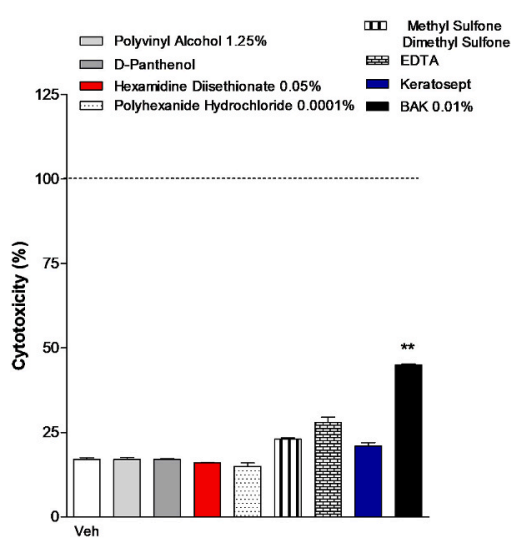

B

10 min exposure

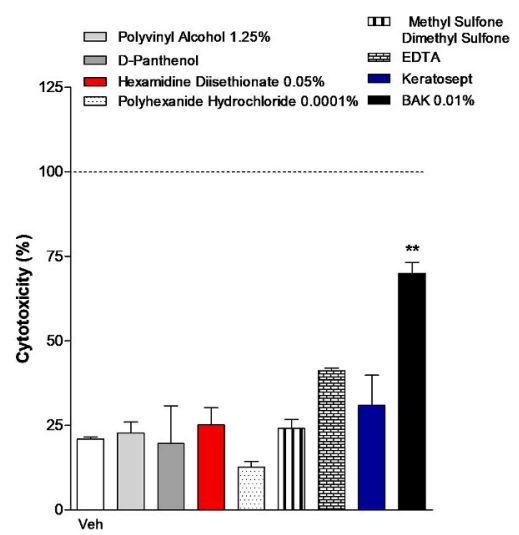

C

\section{5 min exposure}

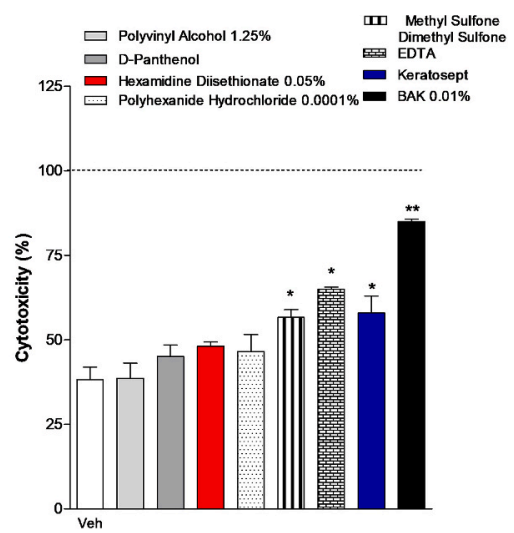

Fig. 2. Evaluation of lactate dehydrogenase (LDH) release in the human corneal epithelial cells (HCE-2) after (A) $5 \mathrm{~min}$, (B) $10 \mathrm{~min}$ or (C) $15 \mathrm{~min}$ of incubation with Keratosept or its components. Data are expressed as percentage of the maximum degree of cell death (incubation of cells for $2 \mathrm{~h}$ with $0.01 \%$ of BAK); they represent the mean \pm standard error of the mean of at least 3 experiments performed in triplicate. * $p<0.05$ vs Veh; **p $<0.01$ vs Veh; Veh $=$ vehicle, EDTA $=$ disodium edetate, $\mathrm{BAK}=$ benzalkonium chloride, $\mathrm{D}$-Panthenol $=$ Dexpanthenol. 
A 5 min exposure

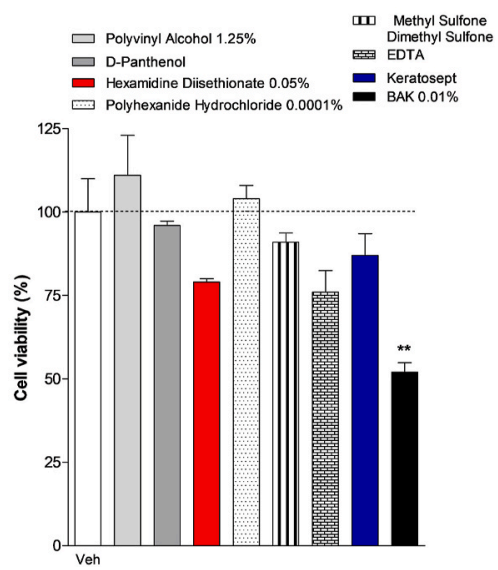

B

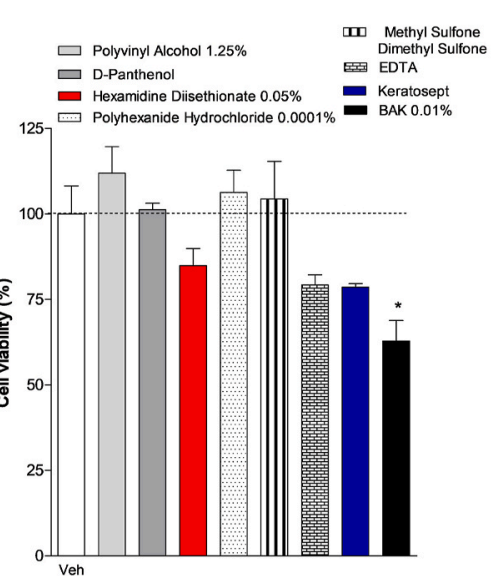

C

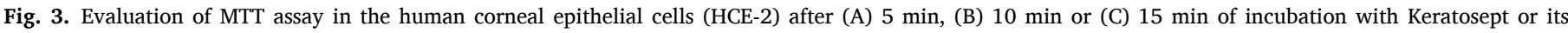

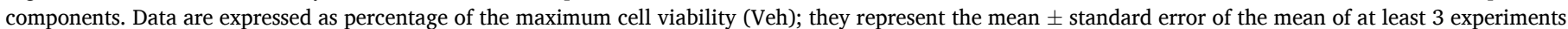

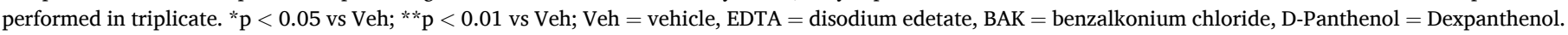

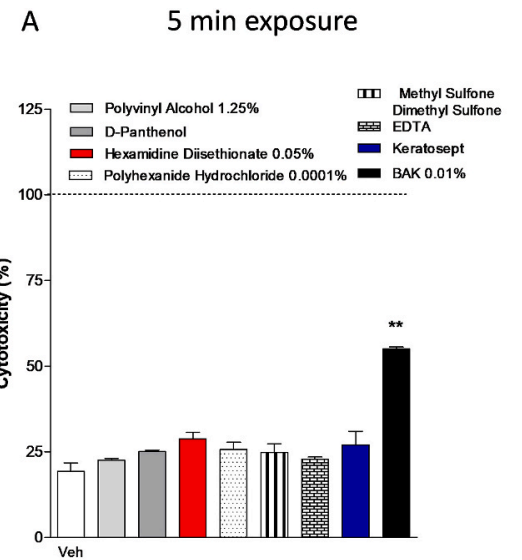

B

10 min exposure

C $15 \mathrm{~min}$ exposure
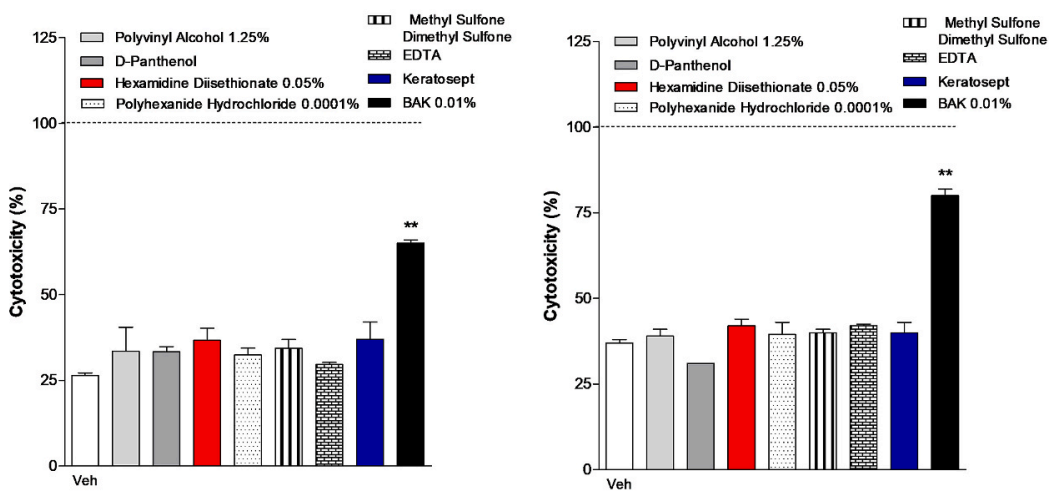

Fig. 4. Evaluation of lactate dehydrogenase (LDH) release in the human conjunctival epithelial cells (HConEC) after (A) $5 \mathrm{~min}$, (B) $10 \mathrm{~min}$ or (C) $15 \mathrm{~min}$ of incubation with Keratosept or its components. Data are expressed as percentage of the maximum degree of cell death (incubation of cells for $2 \mathrm{~h}$ with $0.01 \%$ of BAK); they represent the mean \pm standard error of the mean of at least 3 experiments performed in triplicate. ${ }^{* *} \mathrm{p}<0.01 \mathrm{vs}$ Veh; Veh $=$ vehicle, EDTA $=$ disodium edetate, $\mathrm{BAK}=$ benzalkonium chloride, D-Panthenol $=$ Dexpanthenol.

A

5 min exposure
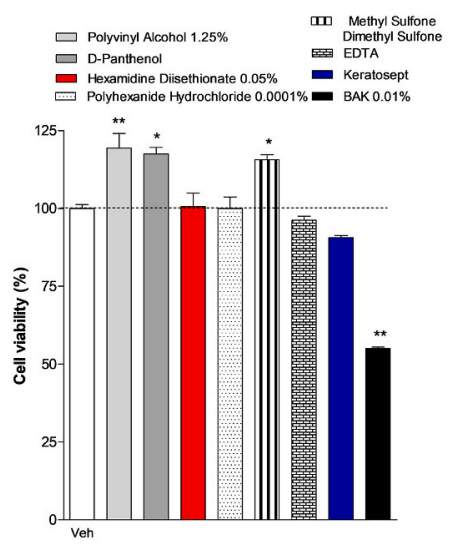

B

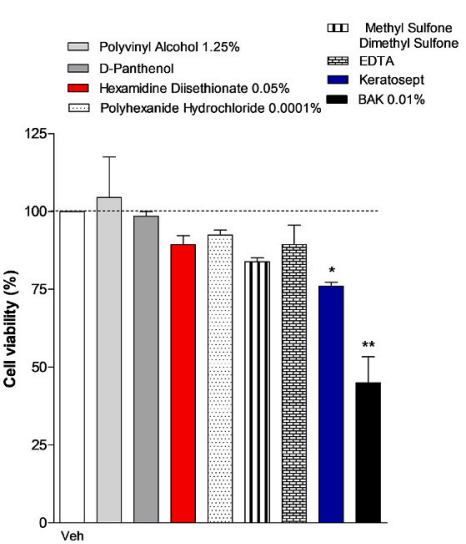

C

$15 \min$ exposure
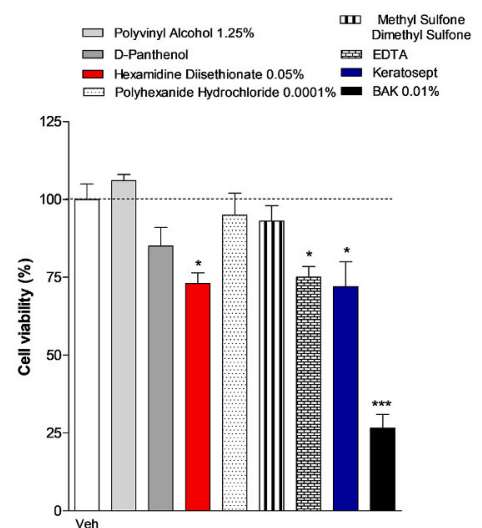

Fig. 5. Evaluation of MTT assay in the human conjunctival epithelial cells (HConEC) after (A) 5 min, (B) 10 min or (C) 15 min of incubation with Keratosept or its components. Data are expressed as percentage of the maximum cell viability (Veh); they represent the mean \pm standard error of the mean of at least 3 experiments performed in triplicate. ${ }^{*} \mathrm{p}<0.05$ vs Veh; ${ }^{* *} \mathrm{p}<0.01$ vs Veh; Veh $=$ vehicle, EDTA = disodium edetate, BAK = benzalkonium chloride, D-Panthenol $=$ Dexpanthenol. 


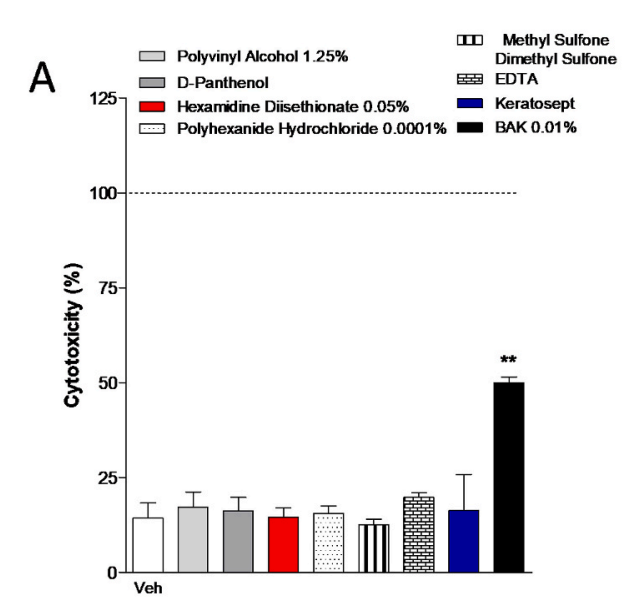

\section{HCE2}
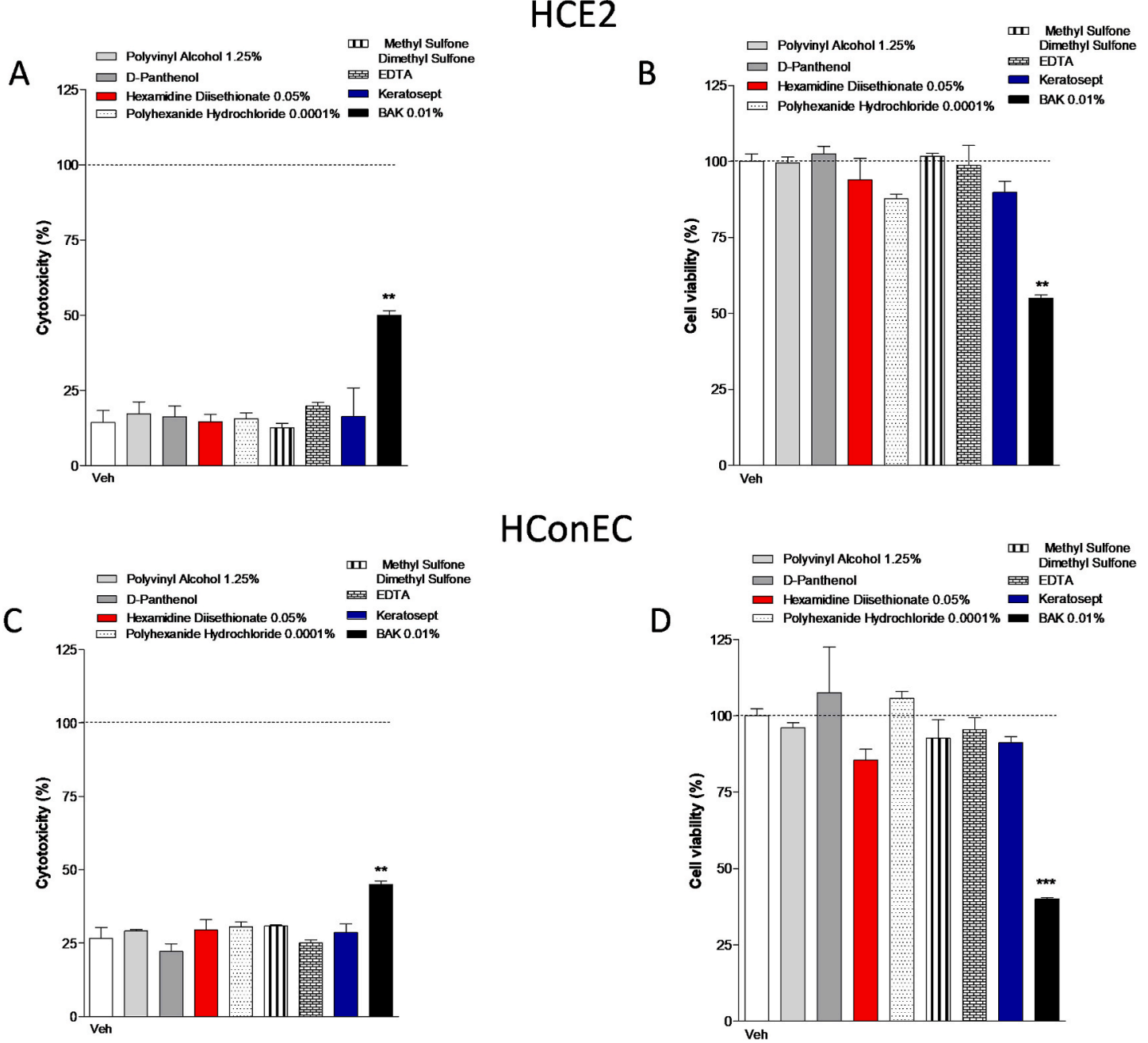

\section{HCOnEC}

D

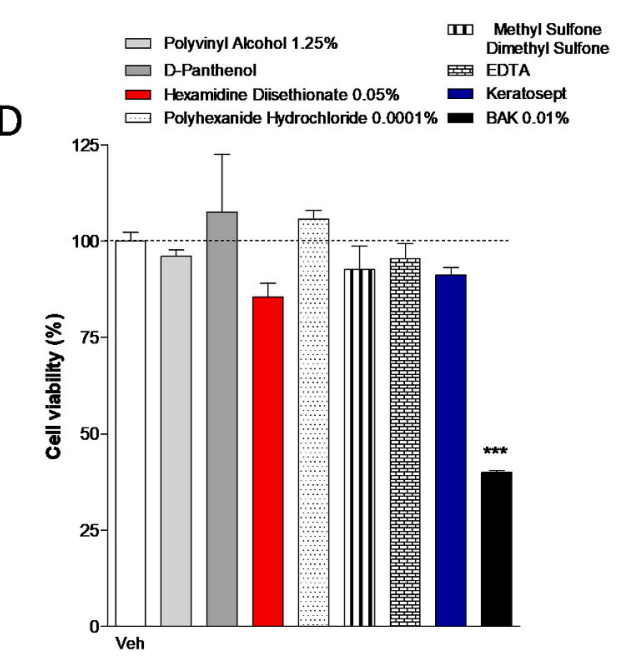

Fig. 6. Evaluation of lactate dehydrogenase (LDH) release in human corneal (HCE-2) (A) and conjunctival (HConEC) (B) epithelial cells and cell viability by MTT assay in corneal (B) or conjunctival (D) cells after $15 \mathrm{~min}$ of incubation with Keratosept or its components at a 1:10 dilution. Data are expressed as percentage of the maximum degree of cell death (incubation of cells for $2 \mathrm{~h}$ with $0.01 \%$ of BAK); they represent the mean \pm standard error of the mean of at least 3 experiments performed in triplicate. ${ }^{* *} \mathrm{p}<0.01$ vs Veh; ${ }^{* * *} \mathrm{p}<0.001$ vs Veh; Veh $=$ vehicle, EDTA $=$ disodium edetate, BAK $=$ benzalkonium chloride, D-Panthenol $=$ Dexpanthenol.

recently, several antiseptic-based ophthalmic formulations have been released into the market.

The ophthalmic solution tested in this study contains 3 antiseptics: PHMB $0.0001 \%$, hexamidine diisethionate $0.05 \%$ and EDTA $0.01 \%$. There are few studies on the antimicrobial activity in vitro and in vivo and the safety of PHMB, as reported by Fjeld and Lingaas (2016). Interestingly, a single study (Hansmann et al., 2004) evaluated the use of PHMB as a preoperative antiseptic for cataract surgery, showing a reduction of the conjunctival flora. Regarding hexamidine diisethionate, alone or in combination with PHMB, different studies have evaluated the efficacy of the compound at a concentration of $0.1 \%$ for the treatment of Acanthamoeba keratitis (Brasseur et al., 1994; Carrijo-Carvalho et al., 2017; Maycock and Jayaswal, 2016; Perrine et al., 1995), while EDTA showed good efficacy as an antibiofilm agent when used stand-alone or in combination with other antimicrobials (Finnegan and Percival, 2015). However, the antiseptic activity of these compounds needs to be better assessed, also because, to our knowledge, no standardized guidelines (EN, ISO) for the evaluation of the antimicrobial activity of ophthalmic solutions exist.

In our study, the combined effect of these compounds caused a reduction of the bacterial load of $2.14 \log$ within $6 \mathrm{~h}$ of exposure to the ophthalmic solution, especially for Fastidious bacteria (S. pneumonia, $S$. mitis and S. pyogenes). According to Pinna et al. (2020), the Keratosept ophthalmic solution showed, using a qualitative method, an antimicrobial activity in vitro against different microorganisms. However, in our study, we have performed a quantitative analysis during an observational period of $6 \mathrm{~h}$ and we considered $100 \mu \mathrm{L}$ of ophthalmic solution, which is equivalent to approximately 2 drops; for this reason we observed, differently from Pinna et al. (2020), who tested a higher volume $(990 \mu \mathrm{L})$ of Keratosept, a slower reduction of bacterial growth during the analysis period. Moreover, when tested on the surface of solid agar medium, the ophthalmic solution caused inhibition of the microbial growth. Only for $P$. aeruginosa was no inhibition observed: indeed, on a solid medium this microorganism is able to form robust biofilms (Lee and Yoon, 2017) and this ability is well-known for the corneal surface and contact lenses as well (Zegans et al., 2002).

Further in vitro and clinical studies are necessary to confirm the antimicrobial efficacy of this solution against different microorganisms, as well as bacterial and fungal biofilms.

Antiseptics such as povidone-iodine may induce cytotoxic effects on the ocular surface epithelial cells, and punctate corneal epithelial keratitis, epithelial defects and conjunctival irritation, as confirmed by in vivo studies (Koerner et al., 2018), especially at high concentrations. Therefore, it is important to assess the potentially harmful effects of antiseptic-containing solutions on the ocular surface.

To our knowledge, no studies have been published on the in vitro 


\section{Control}
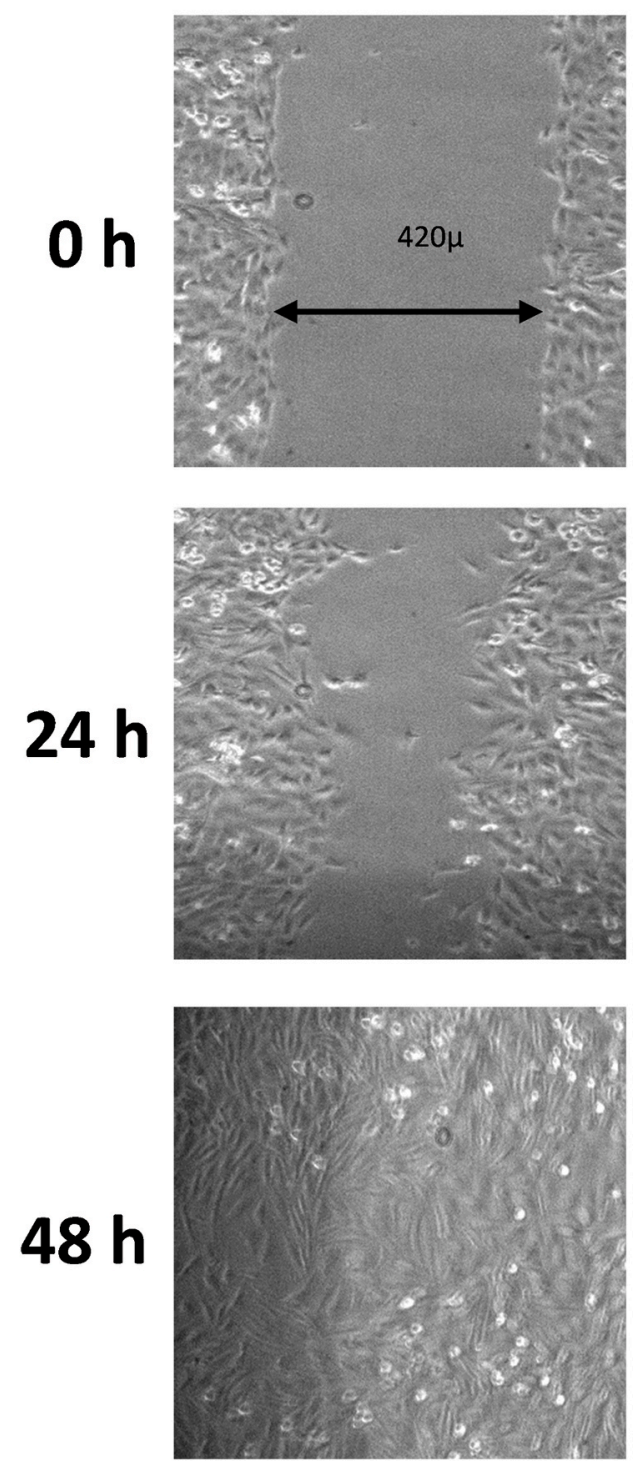

D-Panthenol + Polyvinyl Alcohol
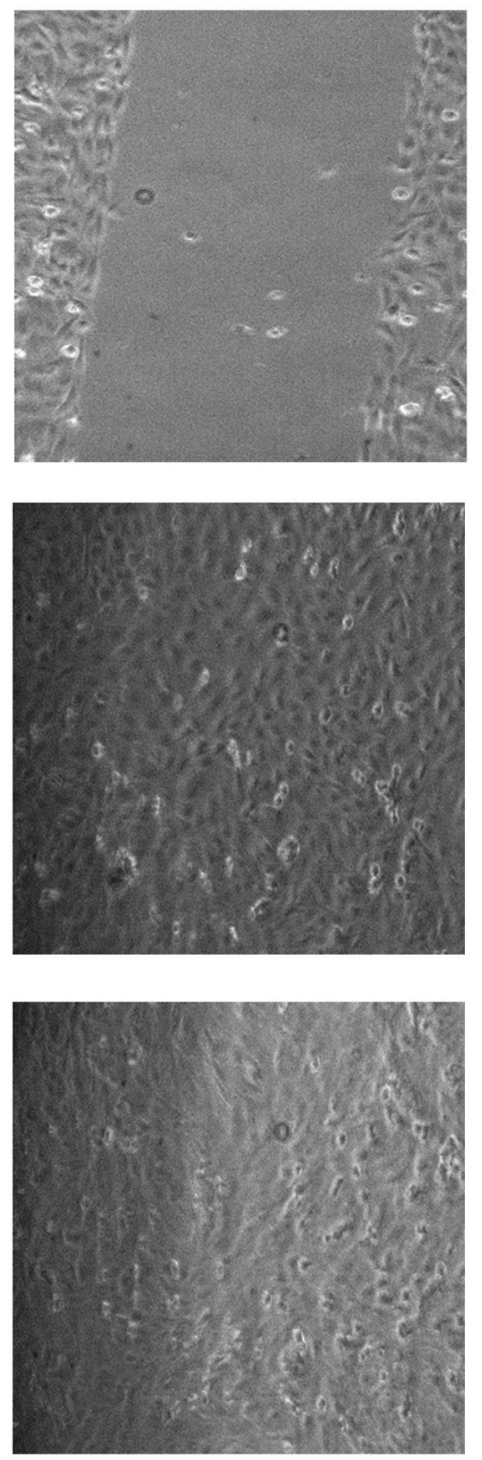

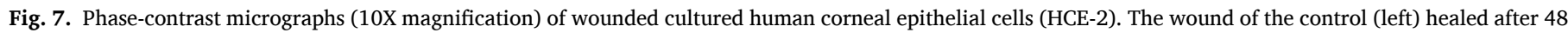
h (bottom left). In cultures exposed to polyvinyl alcohol $1.25 \%$ and D-panthenol 5\% the gap was closed in $24 \mathrm{~h}$ (middle right). D-Panthenol = Dexpanthenol.

effect of Keratosept on cultured conjunctival and corneal epithelial cells up to now. Concerning its components, The Cosmetic Ingredient Review Expert Panel (2007) reported no ocular signs of irritations in rabbits after instillation of hexamidine diisethionate $0.05 \%$ in aqueous solution. PHMB and hexamidine diisethionate toxicity on corneal epithelial cells has been evaluated in few in vitro studies: Shi et al. (2018) reported a reduction in cell proliferation at a maximum concentration of $0.02 \%$ PHMB and of $0.1 \%$ hexamidine. Nevertheless, they used different cell lines and they dissolved the drugs in a culture cell medium: therefore, their results are not directly comparable to our study. Yanai et al. (2006), using a colorimetric method, showed, in cultured human corneal epithelial cells, a low cytotoxicity of PHMB $0.0001 \%$ (1 part per million), which increased in a dose-dependent manner.

In our in vitro studies, exposing human corneal epithelial cells to Keratosept or its components for different periods of time, we found significantly higher cytotoxicity and lower cell viability with Keratosept compared to the vehicle, only after $15 \mathrm{~min}$. Regarding conjunctival epithelial cells the results were similar, with a significant reduction in cell viability after $10 \mathrm{~min}$ with Keratosept, and after $15 \mathrm{~min}$ with some components such as hexamidine, and no increase of cytotoxicity compared to the vehicle in the whole observation period.

These results need to be confirmed by in vivo studies, as the ocular surface system is very different from our experimental setting for various reasons: for example, in a healthy eye the concentration of solutions instilled on the ocular surface can be reduced due to dilution in tears, and the exposure time may be lower due to lacrimal turnover and blinking; moreover, in our experimental setting, the cells were exposed to Keratosept or its components alone, without the nourishment usually supplied by culture medium. Therefore, we tested Keratosept and its components at a 1:10 dilution for 15 min: both the conjunctival and corneal cells did not show any significant cytotoxicity or reduced viability compared to the vehicle. Moreover, in our experimental setting, D-panthenol and polyvinyl alcohol showed an increase in cell viability at $5 \mathrm{~min}$ in human conjunctival epithelial cells and accelerated 
the gap closure between corneal epithelial cells. This is consistent with the wound-healing properties of D-panthenol reported in studies on human dermal fibroblasts (Weimann and Hermann, 1999) and skin wounds (Heise et al., 2012), which explain its wide use in dermatology (Proksch et al., 2017). Polyvinyl alcohol is a biocompatible polymer, often present in ophthalmic solutions as a viscosity-enhancing agent (Ávila-Salas et al., 2019).

In conclusion, in our study Keratosept ophthalmic solution showed a good in vitro antimicrobial activity on the bacterial strains most frequently responsible for ocular infections, including MRSA resistant to fluoroquinolones and aminoglycosides; however, Pseudomonas aeruginosa, if grown on solid agar medium, appeared to be not susceptible. Moreover, Keratosept showed low cytotoxicity on cultured corneal and conjunctival epithelial cells.

However, further in vitro studies are necessary to assess more precisely the antimicrobial efficacy of Keratosept ophthalmic solution, even on bacterial biofilms, and to evaluate the clinical antimicrobial efficacy on ocular surface infections; moreover, the tolerability of Keratosept needs to be confirmed in clinical studies. It could be interesting to compare, with the same experimental settings, the antimicrobial efficacy and the cytotoxicity of different antiseptic-containing ophthalmic solutions.

\section{Funding declaration}

This research did not receive any specific grant from funding agencies in the public, commercial, or not-for-profit sectors.

\section{Declaration of competing interest}

None.

\section{References}

Asbell, P.A., DeCory, H.H., 2018. Antibiotic resistance among bacterial conjunctival pathogens collected in the Antibiotic Resistance Monitoring in Ocular Microorganisms (ARMOR) surveillance study. PloS One 13. https://doi.org/ 10.1371/journal.pone.0205814. eCollection 2018 e0205814.

Ávila-Salas, F., Marican, A., Pinochet, S., Carreño, G., Valdés, O., Venegas, B., Wendy Donoso, W., Cabrera-Barjas, G., Vijayakumar, S., Durán-Lara, E.F., 2019. Film dressings based on hydrogels: simultaneous and sustained-release of bioactive compounds with wound healing properties. Pharmaceutics 11, 447. https://doi.org/ 10.3390/pharmaceutics11090447.

Bertino Jr., J.S., 2009. Impact of antibiotic resistance in the management of ocular infections: the role of current and future antibiotics. Clin. Ophthalmol. 3, 507-521. https://doi.org/10.2147/opth.s5778.

Brasseur, G., Favennec, L., Perrine, D., Chenu, J.P., Brasseur, P., 1994. Successful treatment of Acanthamoeba keratitis by hexamidine. Cornea 13, 459-462. https:// doi.org/10.1097/00003226-199409000-00015.

Carrijo-Carvalho, L.C., Sant'ana, V.P., Foronda, A.S., de Freitas, D., de Souza Carvalho, F. R., 2017. Therapeutic agents and biocides for ocular infections by free-living amoebae of Acanthamoeba genus. Surv. Ophthalmol. 62, 203-218. https://doi.org/ 10.1016/j.survophthal.2016.10.009.

Finnegan, S., Percival, S.L., 2015. EDTA: an antimicrobial and antibiofilm agent for use in wound care. Adv. Wound Care 4, 415-421. https://doi.org/10.1089/ wound.2014.0577.

Fjeld, H., Lingaas, E., 2016. Polyhexanide - safety and efficacy as an antiseptic. Tidsskr. Nor. Laegeforen. 136, 707-711. https://doi.org/10.4045/tidsskr.14.1041.

Garg, P., Roy, A., Sharma, S., 2017. Endophthalmitis after cataract surgery: epidemiology, risk factors, and evidence on protection. Curr. Opin. Ophthalmol. 28 67-72. https://doi.org/10.1097/icu.0000000000000326.

Green, M., Apel, A., Stapleton, F., 2008. Risk factors and causative organisms in microbial keratitis. Cornea 27, 22-27. https://doi.org/10.1097/ ico.0b013e318156caf2.

Grzybowski, A., Brona, P., Kim, S.J., Koerner, J.C., George, M.J., Meyer, D.R., Rosco, M. G., Habib, M.M., 2017. Microbial flora and resistance in ophthalmology: a review. Graefes Arch. Clin. Exp. Ophthalmol. 255, 851-862. https://doi.org/10.1007/ s00417-017-3608-y.

Grzybowski, A., Kanclerz, P., Myers, W.G., 2018. The use of povidone-iodine in ophthalmology. Curr. Opin. Ophthalmol. 29, 19-32. https://doi.org/10.1097/ icu. 0000000000000437.

Han, D.P., Wisniewski, S.R., Wilson, L.A., Barza, M., Vine, A.K., Doft, B.H., Kelsey, S.F., 1996. Spectrum and susceptibilities of microbiologic isolates in the endophthalmiti vitrectomy study. Am. J. Ophthalmol. 122, 1-17. https://doi.org/10.1016/s00029394(14)71959-2.

Hansmann, F., Kramer, A., Ohgke, H., Strobel, H., Müller, M., Geerling, G., 2004. Polyhexamethylbiguanid (PHMB) as preoperative antiseptic for cataract surgery. Ophthalmologe 101 (4), 377-383. https://doi.org/10.1007/s00347-003-0933-9.

Heise, R., Skazik, C., Marquardt, Y., Czaja, K., Sebastian, K., Kurschat, P., Gan, L., Denecke, B., Ekanayake-Bohlig, S., Wilhelm, K.P., Merk, H.F., Baron, J.M., 2012. Dexpanthenol modulates gene expression in skin wound healing in vivo. Skin. Pharmacol. Phys. 25, 241-248. https://doi.org/10.1159/000341144.

Holland, E.J., McDonald, M.B., Parekh, J.G., Sheppard, J.D., 2014. Antibiotic resistance in acute postoperative endophthalmitis. Ophthalmology 121 (11 Suppl. 1), S1-S9. https://doi.org/10.1016/j.ophtha.2014.06.049.

Horner, C., Mawer, D., Wilcox, M., 2012. Reduced susceptibility to chlorhexidine in staphylococci: is it increasing and does it matter? J. Antimicrob. Chemother. 67, 2547-2559. https://doi.org/10.1093/jac/dks284.

International Organization for Standardization ISO, 2001. Ophthalmic optics - contact lens care products - microbiological requirements and test methods for products and regimens for hygienic management of contact lenses. ISO Standard No. 14729. https://www.iso.org/standard/25382.html. (Accessed 18 June 2020).

Isenberg, S.J., Apt, L., Valenton, M., Sharma, S., Garg, P., Thomas, P.A., Parmar, P., Kaliamurthy, J., Reyes, J.M., Ong, D., 2017. Prospective, randomized clinical trial of povidone-iodine $1.25 \%$ solution versus topical antibiotics for treatment of bacterial keratitis. Am. J. Ophthalmol. 176, 244-253. https://doi.org/10.1016/j. ajo.2016.10.004.

Kaehn, K., 2010. Polihexanide: a safe and highly effective biocide. Skin Pharmacol. Physiol. 23 (Suppl. 1), 7-16. https://doi.org/10.1159/000318237.

Kampf, G., 2016. Acquired resistance to chlorhexidine - is it time to establish an 'antiseptic stewardship' initiative? J. Hosp. Infect. 94, 213-227. https://doi.org/ 10.1016/j.jhin.2016.08.018.

Koerner, J.C., George, M.J., Meyer, D.R., Rosco, M.G., Habib, M.M., 2018. Povidoneiodine concentration and dosing in cataract surgery. Surv. Ophthalmol. 63, 862-868. https://doi.org/10.1016/j.survophthal.2018.05.002.

Landucci, E., Pellegrini-Giampietro, D.E., Bilia, A.R., Bergonzi, M.C., 2019. Enhanced neuroprotective effects of panax ginseng $\mathrm{G} 115{ }^{\circledR}$ and ginkgo biloba GK501® combinations in vitro models of excitotoxicity. Int. J. Mol. Sci. 20, 5872. https://doi. org/10.3390/ijms20235872.

Lee, K., Yoon, S.S., 2017. Pseudomonas aeruginosa biofilm, a programmed bacterial life for fitness. J. Microbiol. Biotechnol. 27, 1053-1064. https://doi.org/10.4014/ jmb.1611.11056.

Maycock, N.J., Jayaswal, R., 2016. Update on Acanthamoeba keratitis: diagnosis, treatment, and outcomes. Cornea 35, 713-720. https://doi.org/10.1097/ ICO.0000000000000804.

Perrine, D., Chenu, J.P., Georges, P., Lancelot, J.C., Saturnino, C., Robba, M., 1995. Amoebicidal efficiencies of various diamidines against two strains of Acanthamoeba polyphaga. Antimicrob. Agents Chemother. 39, 339-342. https://doi.org/10.1128/ aac.39.2.339.

Piazzini, V., Landucci, E., D’Ambrosio, M., Tiozzo Fasiolo, L., Cinci, L., Colombo, G., Pellegrini-Giampietro, D.E., Bilia, A.R., Luceri, C., Bergonzi, M.C., 2019. Chitosan coated human serum albumin nanoparticles: a promising strategy for nose-to-brain drug delivery. Int. J. Biol. Macromol. 129, 267-280. https://doi.org/10.1016/j. ijbiomac.2019.02.005.

Pinna, A., Donadu, M.G., Usai, D., Dore, S., Boscia, F., Zanetti, S., 2020. Vitro antimicrobial activity of a new ophthalmic solution containing hexamidine diisethionate $0.05 \%$ (Keratosept). Cornea. https://doi.org/10.1097/ ICO.0000000000002375 (Online ahead of print)

Proksch, E., de Bony, R., Trapp, S., Boudon, S., 2017. Topical use of dexpanthenol: a 70th anniversary article. J. Dermatol. Treat. 28, 766-773. https://doi.org/10.1080/ 09546634.2017.1325310.

Shi, L., Stachon, T., Seitz, B., Wagenpfeil, S., Langenbucher, A., Szentmáry, N., 2018. The effect of antiamoebic agents on viability, proliferation and migration of human epithelial cells, Keratocytes and endothelial cells, in vitro. Curr. Eye Res. 43, 725-733. https://doi.org/10.1080/02713683.2018.1447674.

Shibata, Y., Tanaka, Y., Tomita, T., Taogoshi, T., Kimura, Y., Chikama, T., Kihira, K., 2014. Evaluation of corneal damage caused by iodine preparations using human corneal epithelial cells. Jpn. J. Ophthalmol. 58, 522-527. https://doi.org/10.1007/ s10384-014-0348-y.

Teweldemedhin, M., Gebreyesus, H., Atsbaha, A.H., Asgedom, S.W., Saravanan, M., 2017. Bacterial profile of ocular infections: a systematic review. BMC Ophthalmol. 17, 212. https://doi.org/10.1186/s12886-017-0612-2.

The Cosmetic Ingredient Review Expert Panel, 2007. Final report on the safety assessment of hexamidine and hexamidine diisethionate. Int. J. Toxicol. 26 (Suppl. 3), 79-88. https://doi.org/10.1080/10915810701663168.

Weimann, B.I., Hermann, D., 1999. Studies on wound healing: effects of calcium D -pantothenate on the migration, proliferation and protein synthesis of human dermal fibroblasts in culture. Int. J. Vitam. Nutr. Res. 69, 113-119. https://doi.org/ 10.1024/0300-9831.69.2.113.

Yanai, R., Yamada, N., Ueda, K., Tajiri, M., Matsumoto, T., Kido, K., Nakamura, S., Saito, F., Nishida, T., 2006. Evaluation of povidone-iodine as a disinfectant solution for contact lenses: antimicrobial activity and cytotoxicity for corneal epithelial cells. Contact Lens Anterior Eye 29, 85-91. https://doi.org/10.1016/j.clae.2006.02.006.

Zegans, M.E., Becker, H.I., Budzik, J., O'Toole, G., 2002. The role of bacterial biofilms in ocular infections. DNA Cell Biol. 21, 415-420. https://doi.org/10.1089/ 10445490260099700. 